\title{
ANALOGY BETWEEN THERMAL AND MASS DIFFUSION EFFECTS OF A FREE CONVECTIVE FLOW IN RECTANGULAR ENCLOSURE
}

\author{
Vusala Ambethkar \\ Department of Mathematics \\ Faculty of Mathematical Sciences \\ University of Delhi \\ Delhi-110007, India \\ vambethkar@maths.du.ac.in,vambethkar@gmail.com
}

Received: 2 June 2020; Accepted: 19 September 2020

\begin{abstract}
In this investigation, the analogy between thermal and mass diffusive effects of a free convective flow in a rectangular enclosure is emphasized. The upwind finite volume method is used to discretize the governing equations of the continuity, momentum, energy and mass transfer. The novelty in this exploration is to appropriately modify the well-known Semi-Implicit Method for Pressure-Linked Equations (SIMPLE) algorithm so that it suits to the present problem and thereby, the new flow variables such as the temperature and the concentration are computed. An empirical correlation for the average Sherwood number $(\overline{S h})$ that does not exist in literature is suggested in this work. The average Sherwood numbers for distinct fluids (gases and liquids) are calculated, and mass diffusion effects within the horizontal rectangle are analyzed. The average Nusselt numbers $(\overline{\mathrm{Nu}})$ are calculated for distinct fluids such as liquids $(\operatorname{Pr} \gg 1)$, liquid metals $(\operatorname{Pr} \ll 1)$ and gases $(\operatorname{Pr}<1)$ for different Rayleigh numbers in the range of $3 \times 10^{5} \leq R a_{L} \leq 7 \times 10^{9}$ from relevant empirical correlations existing in the literature. Accordingly, the thermal diffusion effects within the horizontal rectangle are analyzed.
\end{abstract}

MSC 2010: 76R05, 76R10, 76M12, 80A20

Keywords: free convective flow, thermal and mass diffusion effects, rectangular enclosure, average Nusselt and Sherwood numbers, empirical correlations

\section{Introduction}

Research on thermal and mass diffusion of free convection in a rectangular enclosure has drawn the curiosity of a great number of researchers due to its extensive applications such as humidity in air and in buildings, cooling towers, electronic cooling systems, cooling of mini channels in the blades of a modern gas turbine.

Combined free and forced convection of a two-dimensional, steady, incompressible flow in a driven horizontal rectangle was investigated by Alleborn et al. [1]. The impact of thermal boundary conditions on free convective flow in a rectangular 
enclosure was studied numerically by Corcione [2]. Momentum and heat transport in a conjugate free convective flow by using streamline and heatline was numerically visualized by Deng and Tang [3]. Free convective flow in a rectangular enclosure by using a combined temperature scale was numerically studied by Deng et al. [4]. Heat and mass transfer effects of a combined free and forced convective flow in a vertical rectangle was studied by Teamah and Maghlany [5]. Globe and Dropkin [6] have investigated natural convective heat transfer in liquids confined by two horizontal plates heated from below and proposed some empirical correlations for Nusselt numbers for different Rayleigh numbers. Hollands et al. [7] have proposed empirical correlations for the Nusselt number of a free convective air and water flow in a horizontal rectangular region. Hoseinzadeh et al. [8] have presented the solution to continuity and the Navier-Stokes equations by using the Revised Semi-Implicit Method for the Pressure-Linked Equations (SIMPLER) algorithm, and the same has been utilized for solving the square cavity problems. Hoseinzadeh et al. [9] have investigated numerically the rectangular thermal energy storage units with multiple phase change materials. A comparison of analytical and numerical methods of thermal analysis of rectangular cross-section porous fins enclosure was investigated by Hoseinzadeh et al. [10,11]. Hoseinzadeh et al. [12] have investigated numerically the heat transfer of a laminar and turbulent pulsating $\mathrm{Al}_{2} \mathrm{O}_{3}$ /water nanofluid flow. The problem of free convective flow in a rectangular enclosure in the presence of four discrete heaters was investigated by Nithyadevi et al. [13]. Patankar and Spalding [14] have introduced a benchmark method for calculating the flow variables linked to a three-dimensional mass, momentum and energy equations. Saglam et al. [15] have investigated numerically and experimentally the problem of free convection in an enclosure fitted with heat sources. Steady free convective flow in a rectangular region with wall heat and concentration sources was studied by Vusala and Basumatary [16].

Motivation to this current exploration is due to non-existence of mass transfer analysis of a free convective flow in rectangular enclosure, as revealed by the existing literature. Furthermore, for this kind of flow, an appropriate dimensionless number such as the average Sherwood number $(\overline{S h})$ being used for examining the mass transfer is not yet available. Therefore, under these circumstances, the real challenge is to come up with appropriate correlations for the mass transfer dimensionless number and the average Sherwood number. However, for studying the mass transfer effects, no correlations for the average Sherwood number exist in the literature. Therefore, motivation to this current study by this objection and in this study, correlations for the average Sherwood number are suggested. Nevertheless, as per existing literature, for the purpose of the heat transfer study, definite empirical correlations are available for average Nusselt number $(\overline{\mathrm{Nu}})$ of a natural convection in rectangle enclosures. Thus, the numerical solutions of the average Nusselt and Sherwood numbers are computed and the aspects of combined heat and mass transfer of natural convection in rectangular enclosure are studied.

The novelty in this current problem is to properly amend the well-known SemiImplicit Method for Pressure-Linked Equations (SIMPLE) algorithm and thereby, 
we are able to compute the new flow variables such as the temperature $(\theta)$, the concentration $(C)$ in addition to the velocity and the pressure. The other innovations included in the present problem are deriving the discretized energy, mass transfer equations and by analogy, an empirical correlation for average Sherwood number is suggested that enables us to analyze the mass transfer.

The aim of this paper is to discretize the governing equations by using the upwind finite volume scheme. The original SIMPLE algorithm put forth by Patankar and Spalding [14] has been modified appropriately and, consequently, the new flow variables such as the temperature, concentration are computed. An empirical correlation for the average Sherwood number is suggested and thereby the nature of the mass transfer in a horizontal rectangle is analyzed. We calculate the average Nusselt number by using the empirical correlation that exist in the literature [17] and thereby discuss the nature of the heat transfer within the rectangle. The validity of the present numerical solutions obtained from the empirical correlations which are being suggested in this study and as well as from those existing in the literature is guaranteed.

\section{Formulation}

\subsection{Description}

The schematic illustration about free convective flow in a rectangular region with a heat and a mass source placed on its lower wall is shown in Figure 1. Consider a rectangular region denoted by $\mathrm{ABCD}$ that is of length $H$ and height $L$ in which free convective flow is considered. For the purpose of maintaining free convection within ABCD, no-slip boundary conditions for velocity are defined on these walls. Let $T_{h}$ and $T_{c}, c_{h}$ and $c_{c}$ be the temperature and the concentration flow variables defined on the bottom and the top wall. Due to the difference in temperature defined by $T=T_{h}$ and $T=T_{c}$ on the bottom and the top wall, the free convective flow is sustained within the rectangle. Similarly, due to the difference in concentration defined by $c=c_{h}$ and $c=c_{c}$ on the bottom and the top wall, mass diffusion effects prevails within the rectangular region [16].

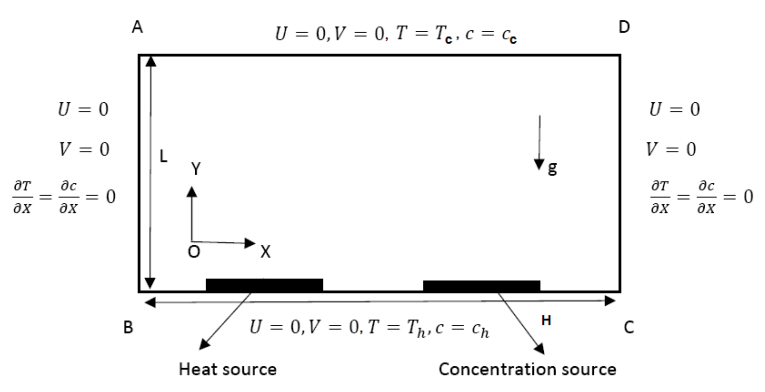

Fig. 1. Schematic of the problem 


\subsection{Governing equations}

Free convective flow with thermal and mass diffusion effects is governed by the equation of continuity, $X$ and $Y$ components of the momentum equation, the energy and the mass transfer equation written in dimensional form as follows [16]:

$$
\begin{array}{ll}
\text { continuity equation: } & \frac{\partial U}{\partial X}+\frac{\partial V}{\partial Y}=0, \\
X \text {-momentum equation: } & U \frac{\partial U}{\partial X}+V \frac{\partial U}{\partial Y}=-\frac{1}{\rho} \frac{\partial p}{\partial X}+v\left(\frac{\partial^{2} U}{\partial X^{2}}+\frac{\partial^{2} U}{\partial Y^{2}}\right), \\
Y \text {-momentum equation: } \quad & U \frac{\partial V}{\partial X}+V \frac{\partial V}{\partial Y}=-\frac{1}{\rho} \frac{\partial p}{\partial Y}+v\left(\frac{\partial^{2} V}{\partial X^{2}}+\frac{\partial^{2} V}{\partial Y^{2}}\right) \\
& +g \beta_{T}\left(T-T_{c}\right)+g \beta_{c}\left(c-c_{c}\right), \\
\text { energy equation: } & U \frac{\partial T}{\partial X}+V \frac{\partial T}{\partial Y}=\alpha\left(\frac{\partial^{2} T}{\partial X^{2}}+\frac{\partial^{2} T}{\partial Y^{2}}\right), \\
\text { mass transfer equation: } \quad & U \frac{\partial c}{\partial X}+V \frac{\partial c}{\partial Y}=D\left(\frac{\partial^{2} c}{\partial X^{2}}+\frac{\partial^{2} c}{\partial Y^{2}}\right)
\end{array}
$$

where $U, V, X, Y, p, T, c, \rho, \alpha, v, g, \beta_{T}, \beta_{c}, T_{c}, c_{c}$ and $D$ are the dimensional velocity components along $X$ and $Y$-directions, independent variables, pressure, temperature, concentration, density, thermal diffusivity, kinematic viscosity, acceleration due to gravity, coefficient of thermal expansion at constant temperature, coefficient of thermal expansion at constant concentration, temperature of the cold wall, concentration of the cold wall and mass diffusivity respectively. We introduce the dimensionless variables as follows [17] and [16]:

$$
\begin{aligned}
& (x, y)=\frac{(X, Y)}{L},(u, v)=\frac{(U, V) L}{v}, P=\frac{p L^{2} \rho}{\mu^{2}}, \theta=\frac{T-T_{c}}{T_{h}-T_{c}}, C=\frac{c-c_{c}}{c_{h}-c_{c}}, \operatorname{Pr}=\frac{v}{\alpha}, \\
& S c=\frac{v}{D}, L e=\frac{S c}{P r}, G r_{T}=\frac{g \beta_{T}\left(T_{h}-T_{c}\right)}{v^{2}} L^{3}, G r_{C}=\frac{g \beta_{c}\left(c_{h}-c_{c}\right)}{v^{2}} L^{3}
\end{aligned}
$$

where $x, y, L, u, v, P, \mu \theta, C, G r_{T}, G r_{c}, P r$ and $L e, S c$ are dimensionless independent variables along $X$ and $Y$ directions, height of the rectangle, dimensionless velocity components along $X$ and $Y$-directions, dimensionless pressure, coefficient of dynamic viscosity, dimensionless temperature, dimensionless concentration, the Grashof number with reference to the temperature and concentration respectively, the Prandtl number, the Lewis number and the Schmidt number. The subscripts $h$, and $c$, represents the hot and cold conditions [17] and [16]. 
The dimensional boundary conditions for the governing equations (1)-(5) are:

$$
\left.\begin{array}{l}
\text { on } \mathrm{AB}: \quad \text { at } X=0, U=V=0, \frac{\partial T}{\partial X}=0, \frac{\partial c}{\partial X}=0, \\
\text { on } \mathrm{DC}: \quad \text { at } X=H, U=V=0, \frac{\partial T}{\partial X}=0, \frac{\partial c}{\partial X}=0, \\
\text { on } \mathrm{BC}: \quad \text { at } Y=0, U=V=0, T=T_{h}, c=c_{h}, \\
\text { on } \mathrm{AD}: \quad \text { at } Y=L, U=0, V=0, T=T_{c}, c=c_{c},
\end{array}\right\}
$$

Where $H$ and $L$ are the length and height of the horizontal rectangle.

Using the dimensionless variables, the governing equations (1)-(5) become as:

$$
\begin{array}{ll}
\text { continuity equation: } & \frac{\partial u}{\partial x}+\frac{\partial v}{\partial y}=0, \\
\text {-momentum equation: } & u \frac{\partial u}{\partial x}+v \frac{\partial u}{\partial y}=-\frac{\partial P}{\partial x}+\left(\frac{\partial^{2} u}{\partial x^{2}}+\frac{\partial^{2} u}{\partial y^{2}}\right), \\
y \text {-momentum equation: } \quad & u \frac{\partial v}{\partial x}+v \frac{\partial v}{\partial y}=-\frac{\partial P}{\partial y}+\left(\frac{\partial^{2} v}{\partial x^{2}}+\frac{\partial^{2} v}{\partial y^{2}}\right) \\
& +G r_{T} \theta+G r_{c} C, \\
\text { energy equation: } & u \frac{\partial \theta}{\partial x}+v \frac{\partial \theta}{\partial y}=\frac{1}{P r}\left(\frac{\partial^{2} \theta}{\partial x^{2}}+\frac{\partial^{2} \theta}{\partial y^{2}}\right), \\
\text { mass transfer equation: } & u \frac{\partial C}{\partial x}+v \frac{\partial C}{\partial y}=\frac{1}{L e P r}\left(\frac{\partial^{2} C}{\partial x^{2}}+\frac{\partial^{2} C}{\partial y^{2}}\right),
\end{array}
$$

where $x, y, u, v, P, \theta, C, G r_{T}, G r_{c}, P r$ and $L e$ are dimensionless independent variables along $X$ and $Y$ directions, velocity components along $x$ and $y$-axis, dimensionless velocity components along $X$ and $Y$-directions, dimensionless pressure, dimensionless temperature, dimensionless concentration, the Grashof number with reference to the temperature and concentration, the Prandtl number and the Lewis number. The subscripts $h$, and $c$, represent the hot and cold conditions [17] and [16].

The dimensionless form of the above boundary conditions reducing to:

$$
\left.\begin{array}{l}
\text { on } \mathrm{AB}: \quad \text { at } x=0, u=v=0, \frac{\partial \theta}{\partial x}=0, \frac{\partial C}{\partial x}=0 \\
\text { on } \mathrm{DC}: \quad \text { at } x=2, u=v=0, \frac{\partial \theta}{\partial x}=0, \frac{\partial C}{\partial x}=0 \\
\text { on } \mathrm{BC}: \quad \text { at } y=0, u=v=0, \theta=1, C=1, \\
\text { on } \mathrm{AD}: \quad \text { at } y=1, u=0, v=0, \theta=0, C=0 .
\end{array}\right\}
$$

where $A B, D C, B C$, and $A D$ are the left, right, bottom and top walls of the rectangle. 


\section{Numerical method}

\subsection{Discretization technique}

We have utilized the upwind finite volume scheme and a staggered grid as given in [18, p. 194-200] and [16] to discretize the governing equations (7)-(11). This scheme is more appropriate here because it satisfies the conservativeness, boundedness and transportiveness properties and hence it gives accurate solutions. Complexities involved in the governing equations such as non-linearities and coupling of pressurevelocity compels us to adopt an iterative solution strategy. Furthermore, in addition to the pressure, two more scalar variables, the temperature and the concentration are appearing due to the energy and mass transfer equations. Hence, we need a unique grid(a staggered grid) that allows us to define all scalar variables at the scalar nodes and the vector variables at the interfaces in horizontal and vertical directions of the control volumes constructed around these scalar nodes. This unique feature of a staggered grid enables us to discretize the governing equations suitably on it and also to evaluate the conductive fluxes and the diffusive conductances of the coefficients in the momentum, energy and mass transfer equations at the interfaces of the control volumes.

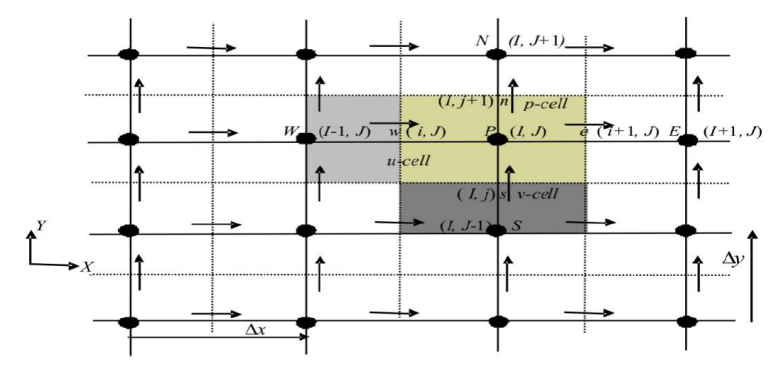

Fig. 2. Staggered grid arrangement

Continuity equation: The discretized continuity equation at location $(I, J)$ is given by [16]

$$
F_{e}-F_{w}+F_{n}-F_{s}=0
$$

where $F_{e}, F_{w}, F_{n}, F_{s}$ are the fluxes across the east, west, north and south cell faces of the control volume.

$x$-momentum: The discretized $u$-momentum equation at location $(i, J)$ is given by

$$
a_{i, J} u_{i, J}=\sum a_{n b} u_{n b}+\left(P_{I-1, J}-P_{I, J}\right) A_{i, J}
$$

where $A_{i, J}$ is the cell face areas of $u$-control volume. $E, W, N, S$ neighbors involved in the summation are $\sum a_{n b} u_{n b}$ are $(i+1, J),(i-1, J),(i, J+1)$ and $(i, J-1)$ in 
accordance with [18, p. 197-199] and [16]. The coefficients of the upwind differencing scheme are

$$
a_{i, J}=a_{i-1, J}+a_{i+1, J}+a_{i, J+1}+a_{i, J-1}+\Delta F
$$

where

$$
\left.\begin{array}{l}
a_{i+1, J}=D_{I, J}+\max \left(-F_{I, J}, 0\right), a_{i-1, J}=D_{I-1, J}+\max \left(F_{I-1, J}, 0\right), \\
a_{i, J+1}=D_{i, j+1}+\max \left(-F_{i, j+1}, 0\right), a_{i, J-1}=D_{i, j}+\max \left(F_{i, j}, 0\right), \\
\Delta F=\left(F_{e}-F_{w}\right)+\left(F_{n}-F_{S}\right)=\left(F_{I, J}-F_{I-1, J}\right)+\left(F_{i, j+1}-F_{i, j}\right)
\end{array}\right\}
$$

The convective flux per unit mass $F$ and the diffusive conductance $D$ for each of the faces $e, w, n$ and $s$ of the $u$-control volume are defined as given in [16, p. 10].

$y$-momentum: The discretized $v$-momentum equation at $(I, j)$ is given by

$$
a_{I, j} v_{I, j}=\sum a_{n b} v_{n b}+\left(P_{I, J-1}-P_{I, J}\right) A_{I, j}+b_{I, j}
$$

where $A_{i, J}$ is the cell face areas of $v$-control volume. The neighbours $E, W, N$ and $S$ involved in the summation $\sum a_{n b} v_{n b}$ are $(I+1, j),(I-1, j),(I, j+1)$ and $(I, j-1)$ in accordance with [18, p. 199-200] and [16].

Now the coefficients of the upwind differencing scheme are as follows:

$$
a_{I, j}=a_{I+1, j}+a_{I-1, j}+a_{I, j+1}+a_{I, j-1}+\Delta F-S_{I, j}
$$

and

$$
S_{I, j} v_{I, j}+b_{I, j}=\bar{S} \Delta v
$$

Similarly, these coefficients contain a combination of the convective flux per unit mass $F$ and the diffusive conductance $D$ for each of the faces $e, w, n$ and $s$ of the $v$-control volume.

The pressure correction equation is given by

$$
a_{I, J} P_{I, J}^{\prime}=a_{I+1, J} P_{I+1, J}^{\prime}+a_{I-1, J} P_{I-1, J}^{\prime}+a_{I, J+1} P_{I, J+1}^{\prime}+a_{I, J-1} P_{I, J-1}^{\prime}+b_{I, J}^{\prime}
$$

where

$$
a_{I, J}=a_{I+1, J}+a_{I-1, J}+a_{I, J+1}+a_{I, J-1}
$$

and the coefficients are

$$
\left.\begin{array}{l}
a_{I+1, J}=(d A)_{i+1, J}, \quad a_{I-1, J}=(d A)_{i, J}, \\
a_{I, J+1}=(d A)_{I, j+1}, \quad a_{I, J-1}=(d A)_{I, j}, \\
d_{i, J}=\frac{A_{i, J}}{a_{i, J}}, \quad d_{I, j}=\frac{A_{I, j}}{a_{I, j}} \\
b_{I, J}^{\prime}=\left(u^{*} A\right)_{i, J}-\left(u^{*} A\right)_{i+1, J}+\left(v^{*} A\right)_{I, j}-\left(v^{*} A\right)_{I, j+1}
\end{array}\right\}
$$


The equation (20) represents the discretized continuity equation evaluated in terms of pressure correction $P^{\prime}$ in accordance with [18, p. 202] and [16]. There is a continuity imbalance in the source term $b^{\prime}$ in this equation due to utilization of the guessed velocities $u^{*}$ and $v^{*}$.

Energy equation: The discretized energy equation at $(I, J)$ is given by [16]

$$
a_{I, J} \theta_{I, J}=\sum a_{n b} \theta_{n b}
$$

The coefficients of the upwind differencing scheme are

$$
a_{I, J}=a_{I+1, J}+a_{I-1, J}+a_{I, J+1}+a_{I, J-1}+\Delta F
$$

These coefficients contain a combination of the convective flux per unit mass $F$ and the diffusive conductance $D$ for each of the faces $e, w, n$ and $s$ of the $p$-control volume are defined in [16, p. 11].

Mass transfer equation: The discretized mass transfer equation at $(I, J)$ is given by [16]

$$
a_{I, J} C_{I, J}=\sum a_{n b} C_{n b}
$$

The coefficients of the upwind differencing scheme are

$$
a_{I, J}=a_{I+1, J}+a_{I-1, J}+a_{I, J+1}+a_{I, J-1}+\Delta F
$$

These coefficients contain a combination of the convective flux per unit mass $F$ and the diffusive conductance $D$ for each of the faces $e, w, n$ and $s$ of the $p$-control volume are defined in [16, p. 11].

\subsection{Numerical calculations}

The real difficulty in the calculation of the velocity field lies in the unknown pressure field. There is no separate equation for determining the pressure variable except the pressure term in gradient form in the momentum equations. Other difficulties in the governing equations is non-linearity in the momentum equations, coupling of pressure-velocity which introduces a constraint in the solution of the flow field. Furthermore, in addition to the pressure, two more scalar variables, the temperature and the concentration appear due to the energy and mass transfer equations. A remedy for such difficulties associated with the governing equations as mentioned above is to adopt an iterative solution procedure such as the Semi-Implicit Method for PressureLinked equation (SIMPLE) algorithm in modified form for determining the numerical solutions of flow variables $u, v, P, \theta$ and $C$ iteratively. We have accomplished this with the help of an improved Semi-Implicit Method for Pressure-Linked Equations (SIMPLE) algorithm and a code executed in C. 


\section{Improved SIMPLE algorithm}

The well-known Semi-Implicit Method for Pressure-Linked Equations (SIMPLE) algorithm [18, p. 204] has been improved appropriately here and by that, we have computed the temperature and the concentration variables in addition to the other flow variables such as the velocity and the pressure. A summary of this improved algorithm is given below [16]:

Step 1 Start with guessed velocities $\left(u^{*}, v^{*}\right)$ pressure field $P^{*}$, temperature $\theta^{*}$ and concentration $C^{*}$.

Step 2 Calculate the coefficients in the momentum equation, solve discretized momentum equations.

Step 3 Calculate the coefficients of pressure equation, solve pressure correction equations.

Step 4 Correct pressure and velocities:

$$
\left.\begin{array}{l}
P_{I, J}=P_{I, J}^{*}+P_{I, J}^{\prime}, \\
u_{i, J}=u_{i, J}^{*}+d_{i, J}\left(P_{I-1, J}^{\prime}-P_{I, J}^{\prime}\right), \\
v_{I, j}=v_{I, j}^{*}+d_{I, j}\left(P_{I, J-1}^{\prime}-P_{I, J}^{\prime}\right) .
\end{array}\right\}
$$

Step 5 Solve temperature and concentration discretized equations.

Step 6 Replace the previous intermediate values of pressure, velocity, temperature and concentration $\left(P^{*}, u^{*}, v^{*}, \theta^{*}, C^{*}\right)$ with the corrected values $(P, u, v, \theta, C)$, return to Step 2 and repeat this process until the solution converges.

\section{Analysis of results}

In the present investigation, our chief concern is to suggest correlations for the average Sherwood numbers and thereby, we are capable of analyzing the nature of the mass diffusion effects within the rectangular enclosure. Moreover, as the title of this present study indicates, we ought to explore the nature of the heat diffusion effects within the rectangular enclosure. We have accomplished this due to a definite empirical correlation available for the average Nusselt number in the literature. As we have stated in the preceding section, the well-known Semi-Implicit Method for Pressure-Linked Equations (SIMPLE) algorithm [18, p. 204] has been improved appropriately here and by that, we could compute the temperature and the concentration variables in addition to the other flow variables such as the velocity and the pressure. Nevertheless, we are not describing here the profiles of these flow variables due to its less significance, as it is not our main target in this study.

As we have pronounced in the abstract of this study, our target is to determine the total heat transfer from the lower wall of the rectangular region as shown in Figure 1 , and is determined from the average Nusselt number $\overline{N u}$ which is defined as 
$\overline{N u}=\frac{\bar{h} L}{k}$, where $\bar{h}$ is the average convection coefficient and $k$ is the thermal conduction. The average Nusselt number represents the overall convection heat transfer occurring at the entire bottom wall of the rectangular enclosure. The average Nusselt numbers $\overline{N u}$ for these fluids are calculated by using the empirical correlations $\overline{N u}=0.069\left(R a_{L}\right)^{\frac{1}{3}} \operatorname{Pr}^{0.074} ; 3 \times 10^{5} \leq R a_{L} \leq 7 \times 10^{9}$ for free convective heat transfer in horizontal rectangular enclosures as proposed by Hollands et al. [7]. We have calculated the average Nusselt numbers $(\overline{\mathrm{Nu}})$ for distinct fluids such as liquids $(\operatorname{Pr} \gg 1)$, liquid metals $(\operatorname{Pr} \ll 1)$ and gases $(P r<1)$ for different Rayleigh numbers in the range of $3 \times 10^{5} \leq R a_{L} \leq 7 \times 10^{9}$, where $R a_{L}$ is defined as $R a_{L}=G r_{L} P r$ is the heat transfer Rayleigh number. The Prandtl number (Pr) is used to measure the relative effectiveness of momentum and thermal energy transport by diffusion in the velocity and thermal boundary layers. These average Nusselt numbers for liquids, liquid metals and gases obtained are sketched in Figure 3 which is given below. The average Nusselt numbers for liquids $(\operatorname{Pr} \gg 1)$ such as water $(\operatorname{Pr}=4.34)$, engine oil $(P r=47,000)$ and Glycerine $(P r=87,000)$ are calculated for different Rayleigh numbers in the range of $3 \times 10^{5} \leq R a_{L} \leq 7 \times 10^{9}$ and are sketched in Figure 3a. From this figure, it is observed that for a particular liquid such as water $(\operatorname{Pr}=4.34)$, as the Rayleigh number increases in the range of $3 \times 10^{5} \leq R a_{L} \leq$ $7 \times 10^{9}$, the average Nusselt number increases uniformly. Similarly, for the other two liquids such as engine oil $(P r=47,000)$ and Glycerine $(\operatorname{Pr}=87,000)$, as the Rayleigh number increases in the range of $3 \times 10^{5} \leq R a_{L} \leq 7 \times 10^{9}$, the average Nusselt number increases uniformly. Furthermore, from Figure $3 \mathrm{a}$, it is observed that at any particular Rayleigh number in the range of $3 \times 10^{5} \leq R a_{L} \leq 7 \times 10^{9}$, as the Prandtl number increases for these liquids, the average Nusselt number increases uniformly. Thus, the rate of heat diffusion from the bottom wall to the top wall of the rectangular region containing a free convective flow increases for different liquids such as water $(\operatorname{Pr}=4.34)$, engine oil $(\operatorname{Pr}=47,000)$ and Glycerine $(\operatorname{Pr}=87,000)$.

The average Nusselt numbers for liquid metals $(P r \ll 1)$ such as sodium $(P r=$ $=0.0066)$, potassium $(P r=0.0083)$, lead $(P r=0.0110)$, bismuth $(P r=0.0142)$ and mercury $(\operatorname{Pr}=0.029)$ are calculated for different Rayleigh numbers in the range of $3 \times 10^{5} \leq R a_{L} \leq 7 \times 10^{9}$ and are sketched in Figure $3 \mathrm{~b}$. From this figure, it is observed that for any particular liquid metal among sodium $(P r=0.0066)$, potassium $(P r=$ $=0.0083)$, lead $(\operatorname{Pr}=0.0110)$, bismuth $(\operatorname{Pr}=0.0142)$ and mercury $(\operatorname{Pr}=0.029)$, as the Rayleigh number increases in the range of $3 \times 10^{5} \leq R a_{L} \leq 7 \times 10^{9}$, the average Nusselt number increases uniformly. Furthermore, from Figure $3 b$, it is observed that, at any particular Rayleigh number in the range of $3 \times 10^{5} \leq R a_{L} \leq 7 \times 10^{9}$, as the Prandtl number increases for these liquid metals, the average Nusselt number increases uniformly. For gases $(P r<1)$ such as hydrogen, water vapour, oxygen and carbon dioxide, the thermal diffusivity dominates than the momentum diffusivity. The average Nusselt numbers for gases $(\operatorname{Pr}<1)$ such as hydrogen $(\operatorname{Pr}=0.684)$, oxygen $(P r=0.733)$, carbon dioxide $(P r=0.76)$ and air $(P r=0.7869)$ are calculated for different Rayleigh numbers in the range of $3 \times 10^{5} \leq R a_{L} \leq 7 \times 10^{9}$ 


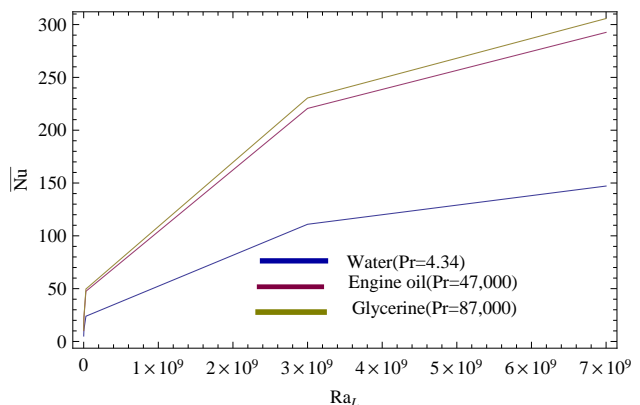

(a) Liquid $(\operatorname{Pr} \gg 1)$

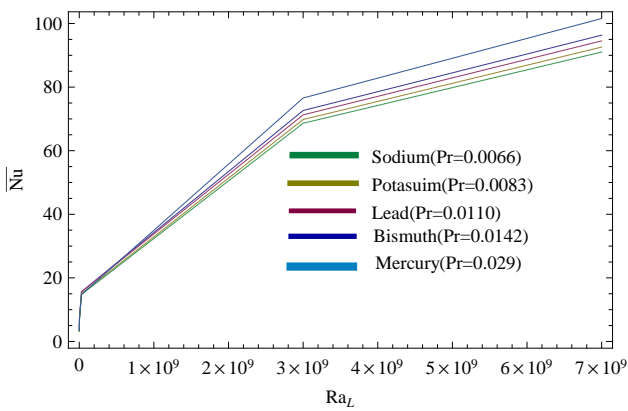

(b) Liquid metals $(P r \ll 1)$

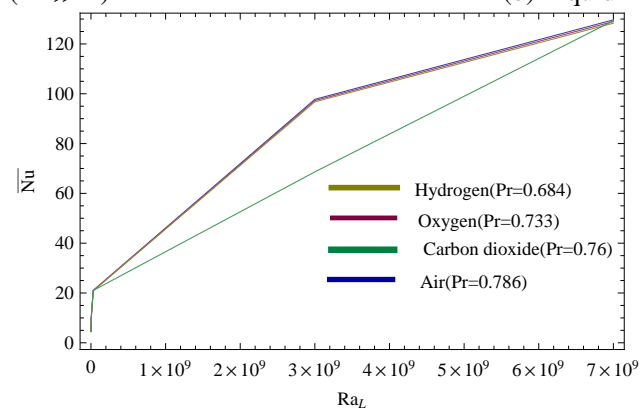

(c) Gases $(\operatorname{Pr}<1)$

Fig. 3. Average Nusselt number for liquids, liquid metals and gases

and are sketched in Figure 3c. From this figure, it is observed that for any particular gas among hydrogen $(P r=0.684)$, oxygen $(P r=0.733)$, carbon dioxide $(P r=0.76)$ and air $(P r=0.7869)$, as the Rayleigh number increases in the range of $3 \times 10^{5} \leq R a_{L} \leq 7 \times 10^{9}$, the average Nusselt number increases uniformly. Furthermore, from Figure $3 \mathrm{c}$, it is observed that, at any particular Rayleigh number in the range of $3 \times 10^{5} \leq R a_{L} \leq 7 \times 10^{9}$, as the Prandtl number increases for these gases, the average Nusselt number increases uniformly. Therefore, the rate of heat diffusion increases from the bottom wall to the top wall of the rectangular region for different liquid metals and gases as mentioned above.

As we have declared in the beginning that our target and contribution in this study is to ascertain the total mass transfer from the lower wall of the rectangular region and is evaluated from the average Sherwood number $\overline{S h}$ which is defined as $\overline{S h_{L}}=\frac{\overline{h_{m}} L}{D}$, where $\overline{h_{m}}$ is the average convection mass transfer coefficient and $D$ is the mass diffusivity. We are able to accomplish all this due to the analogy of empirical correlations persisting among the combined thermal and mass diffusive effects of a free convective flow. The average Sherwood number represents the overall convection mass transfer that occurs at the entire bottom wall of the rectangular enclosure. The average Sherwood numbers $\overline{S h}$ for distinct fluids such as gases $(S c \gg 1$ and $S c<1)$ and liquids $(S c>1)$ are calculated by using the empirical correlation suggested by the present author in this study which is $\overline{S h_{L}}=0.069\left(R a_{m, L}\right)^{\frac{1}{3}} S c^{0.074} ; 3 \times 10^{5} \leq R a_{m, L} \leq 7 \times 10^{9}$, 
where $R a_{m, L}=G r_{c} S c$ is the mass transfer Rayleigh number. By using this correlation, we have calculated the average Sherwood numbers $\overline{S h}$ for these fluids at different Rayleigh numbers in the range of $3 \times 10^{5} \leq R a_{m, L} \leq 7 \times 10^{9}$. We have appropriately chosen the Schmidt numbers $(S c)$ for the species of different gases and liquids mentioned below at a low concentration of $1 \mathrm{~atm}$ and $25^{\circ} \mathrm{C}$ (approx.) and obtained the values of the average Sherwood numbers which are sketched in Figure 4 as given below. The average Sherwood numbers for gases $(S c \gg 1)$ such as hydrogen $(S c=152)$, oxygen $(S c=356)$ and carbon dioxide $(S c=453)$ are calculated for different Rayleigh numbers in the range of $3 \times 10^{5} \leq R a_{m, L} \leq 7 \times 10^{9}$ and are sketched in Figure 4a. It is observed from this figure that at two Rayleigh numbers like $3 \times 10^{9}$ and $7 \times 10^{9}$, for a particular gas such as hydrogen $(S c=152)$, the average Sherwood number increases, and the same is true even for any of the Rayleigh number in the range of $3 \times 10^{5} \leq R a_{m, L} \leq 7 \times 10^{9}$. Similarly, at two Rayleigh numbers like $3 \times 10^{9}$ and $7 \times 10^{9}$, for the other two gases like oxygen $(S c=356)$ and carbon dioxide $(S c=453)$, the average Sherwood number increases uniformly, and the same is true even for any of the Rayleigh numbers in the range of $3 \times 10^{5} \leq R a_{m, L} \leq 7 \times 10^{9}$. Furthermore, from Figure 4a, it is observed that at any particular Rayleigh number among $3 \times 10^{5} \leq R a_{m, L} \leq 7 \times 10^{9}$, as the Schmidt number increases among these gases, the average Sherwood number increases uniformly. Hence, it is concluded that the rate of mass diffusion from the bottom wall to the top wall of the rectangular region containing a free convective flow increases for different gases such as those mentioned above.

The average Sherwood numbers $\overline{S h}$ for gases $(S c<1)$ for different Rayleigh numbers in the range of $3 \times 10^{5} \leq R a_{L} \leq 7 \times 10^{9}$ are calculated by using the empirical correlation suggested by the present author in the above-mentioned paragraph. The Schmidt number $(S c)$ is used to measure the relative effectiveness of momentum and mass transport by diffusion in the velocity and concentration boundary layers. For a different species at low concentration in gases $(S c<1)$ such as hydrogen, water vapour, oxygen and carbon dioxide, the mass diffusivity dominates the momentum diffusivity. The average Sherwood numbers for gases $(S c<1)$ such as hydrogen $(S c=0.22)$, water vapour $(S c=0.60)$ oxygen $(S c=0.75)$ and carbon dioxide $(S c=0.94)$ are calculated for different Rayleigh numbers in the range of $3 \times 10^{5} \leq R a_{L} \leq 7 \times 10^{9}$ and are sketched in Figure $4 \mathrm{~b}$. It is observed from this figure that at two Rayleigh numbers like $3 \times 10^{9}$ and $7 \times 10^{9}$, for a particular gas such as hydrogen $(S c=152)$, the average Sherwood number increases and the same is true even for any of the Rayleigh number in the range of $3 \times 10^{5} \leq R a_{m, L} \leq 7 \times 10^{9}$. Similarly, at two Rayleigh numbers like $3 \times 10^{9}$ and $7 \times 10^{9}$, for other gases such as water vapour $(S c=0.60)$, oxygen $(S c=0.75)$ and carbon dioxide $(S c=0.94)$, the average Sherwood number increases uniformly and the same is true even for any of the Rayleigh numbers in the range of $3 \times 10^{5} \leq R a_{m, L} \leq 7 \times 10^{9}$. Furthermore, from Figure $4 \mathrm{~b}$, it is observed that, at any particular Rayleigh number among $3 \times 10^{5} \leq R a_{m, L} \leq 7 \times 10^{9}$, as the Schmidt number increases among these gases, the average Sherwood number increases uniformly. Hence, it is concluded that the 


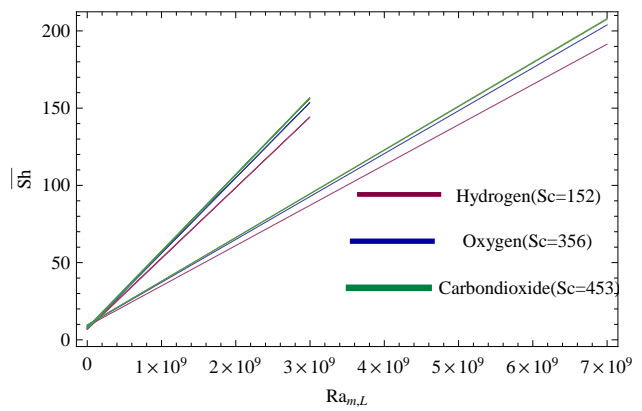

(a) Gases $(S c \gg 1)$

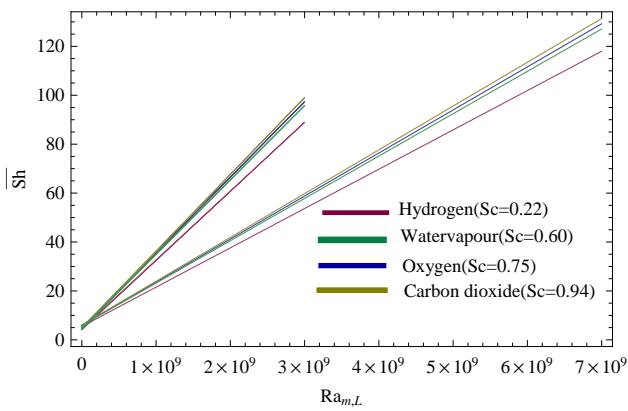

(b) Gases $(S c<1)$

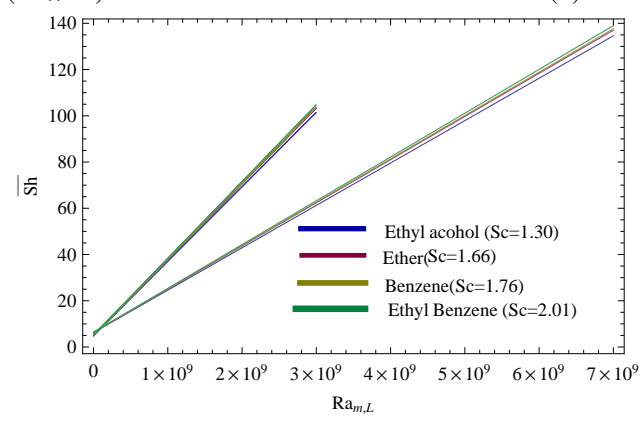

(c) Liquids $(S c>1)$

Fig. 4. Average Sherwood numbers for gases and liquids

rate of mass diffusion from the bottom wall to the top wall of the rectangular region increases for different gases such as those mentioned above.

Analogously, the average Sherwood numbers $\overline{S h}$ for liquids $(S c>1)$ such as ethyl alcohol $(S c=1.30)$, ether $(S c=1.66)$, benzene $(S c=1.76)$ and ethyl benzene $(S c=$ $=2.01$ ) for different Rayleigh numbers in the range of $3 \times 10^{5} \leq R a_{m, L} \leq 7 \times 10^{9}$ are calculated and are sketched in Figure 4c. The momentum diffusivity dominates the mass diffusivity for different species at low concentration in liquids $(S c>1)$ such as ethyl alcohol, ether, benzene and ethyl benzene. From Figure 4c, it is observed that at two Rayleigh numbers like $3 \times 10^{9}$ and $7 \times 10^{9}$, for a particular liquid such as ethyl alcohol $(S c=1.30)$, the average Sherwood number increases, and the same is true even for any of the Rayleigh numbers in the range of $3 \times 10^{5} \leq R a_{m, L} \leq 7 \times 10^{9}$. Similarly, at two Rayleigh numbers like $3 \times 10^{9}$ and $7 \times 10^{9}$, for any other liquids such as ether $(S c=1.66)$, benzene $(S c=1.76)$ and ethyl benzene $(S c=2.01)$, the average Sherwood number increases uniformly and the same is true even for any of the Rayleigh number in the range of $3 \times 10^{5} \leq R a_{m, L} \leq 7 \times 10^{9}$. Furthermore, from Figure $4 \mathrm{c}$, it is observed that at any particular Rayleigh number as $3 \times 10^{9}, 7 \times 10^{9}$ or else among $3 \times 10^{5} \leq R a_{m, L} \leq 7 \times 10^{9}$, as the Schmidt number increases among these liquids, the average Sherwood number increases uniformly. Thus, the rate of mass diffusion from the bottom wall of the rectangular region increases for different 
liquids such as ethyl alcohol, ether, benzene and ethyl benzene for different Rayleigh numbers in the range of $3 \times 10^{5} \leq R a_{m, L} \leq 7 \times 10^{9}$.

\section{Conclusions}

In this paper, empirical correlations for the average Sherwood numbers $(\overline{S h})$ are suggested and consequently, the nature of the mass diffusion effects within the concentration boundary layer is analyzed. The average Sherwood number represents the overall convection mass transfer occurs at the entire bottom wall of the rectangular enclosure. The Schmidt number $(S c)$ is used to measure the relative effectiveness of momentum and mass transport by diffusion in the velocity and concentration boundary layers. The mass diffusivity dominates the momentum diffusivity for different species at low concentration in gases $(S c<1)$ such as hydrogen, water vapour, oxygen and carbon dioxide. Therefore, from the calculated average Sherwood numbers proposed in this study, it is concluded that the rate of mass diffusion from the bottom wall to the top wall of the rectangular region for these gases increases for different mass transfer Rayleigh numbers in the range of $3 \times 10^{5} \leq R a_{m, L} \leq 7 \times 10^{9}$. Analogously, the momentum diffusivity dominates the mass diffusivity for different species at low concentration in liquids $(S c>1)$ such as ethyl alcohol, ether, benzene and ethyl benzene. Therefore, from the calculated average sherwood numbers proposed in this study, it is concluded that the rate of mass diffusion from the bottom wall to the top wall of the rectangular region for these liquids increases for different mass transfer Rayleigh numbers in the range of $3 \times 10^{5} \leq R a_{m, L} \leq 7 \times 10^{9}$. This behaviour could also be seen as the Schmidt number increases among liquids, the average Sherwood number increases uniformly and hence the rate of mass diffusion from the bottom wall to the top wall of the rectangular region increases.

The nature of the thermal diffusion effects in the velocity boundary layer of a free convective flow within the horizontal rectangle is analyzed by making use of the existing empirical correlations for the average Nusselt numbers $(\overline{\mathrm{Nu}})$ which are mentioned in the preceding discussion. The average Nusselt number represents the overall convection heat transfer occurring at the entire bottom wall of the rectangular enclosure. The Prandtl number $(P r)$ is used to measure the relative effectiveness of momentum and thermal energy transport by diffusion in the velocity and thermal boundary layers. The thermal diffusivity dominates the momentum diffusivity in gases $(\operatorname{Pr}<1)$ such as hydrogen, water vapour, oxygen and carbon dioxide. Hence, from the calculated average Nusselt numbers, it is concluded that the thermal diffusion effects from the bottom wall to the top wall of the rectangular region for these gases increases for different heat transfer Rayleigh numbers in the range of $3 \times 10^{5} \leq R a_{L} \leq 7 \times 10^{9}$. Analogously, the momentum diffusivity dominates the thermal diffusivity in liquids $(\operatorname{Pr}>1)$ such as water, engine oil and glycerine. Hence, from the calculated average Nusselt numbers, it is concluded that the thermal diffusion effects from the bottom wall to the top wall of the rectangular region for these liquids increases for different 
heat transfer Rayleigh numbers in the range of $3 \times 10^{5} \leq R a_{L} \leq 7 \times 10^{9}$. Furthermore, the thermal diffusivity dominates the momentum diffusivity for any particular liquid metal $(P r \ll 1)$ such as sodium potassium, lead, bismuth and mercury. The effect of momentum diffusivity is almost negligible on the thermal diffusivity. Consequently, the calculated average Nusselt numbers are observed to increase uniformly and therefore, thermal diffusion effects from the bottom wall to the top wall of the rectangular region increases for these liquids metals as the Rayleigh number increases in the range of $3 \times 10^{5} \leq R a_{L} \leq 7 \times 10^{9}$.

\section{References}

[1] Allenborn, N., Raszillier, H., \& Durst, F. (1999). Lid-driven cavity with heat and mass transport. International Journal of Heat and Mass Transfer, 42, 833-853.

[2] Corcione, M. (2003). Effects of the thermal boundary conditions at the side walls upon natural convection in rectangular enclosures heated from below and cooled from above. International Journal of Thermal Sciences, 42, 199-208.

[3] Deng, Q.-H., \& Tang, G.-F. (2002). Numerical visualization of mass and heat transport for conjugate natural convection/heat conduction by streamline and heatline. International Journal of Heat and Mass Transfer, 45, 2373-2385.

[4] Deng, Q.-H., Tang, G.-F., \& Li, Y. (2002). A combined temperature scale for analyzing natural convection in rectangular enclosures with discrete wall heat sources. International Journal of Heat and Mass Transfer, 45, 3437-3446.

[5] Teamah, M.A., \& Maghlany, M.E. (2010). Numerical simulation of double-diffusive mixed convective flow in rectangular enclosure with insulated moving lid. International Journal of Thermal Sciences, 49, 1625-1638.

[6] Globe, S., \& Dropkin, D. (1959). Natural convection heat transfer in liquids confined by two horizontal plates and heated from below. Journal of Heat Transfer, 81, 24-28.

[7] Hollands, K.G.T., Raithby, G.D, \& Konicek, L. (1975). Correlation equations for free convection heat transfer in horizontal layers of air and water. International Journal of Heat and Mass Transfer, 18, 879-884.

[8] Hoseinzadeh, S., Ostadhossein, R., Mirshahvalad, H.R., \& Seraj, J. (2017). Using SIMPLER algorithm for cavity flow problem. Mechatronics and Applications: An International Journal (MECHATROJ), 1, 55-63.

[9] Hoseinzadeh, S., Ghasemiasl, R., Havaei, A., \& Chamkha, A.J. (2018). Numerical investigation of rectangular thermal energy storage units with multiple phase change materials. Journal of Molecular Liquids, 271, 655-660.

[10] Hoseinzadeh, S., Moafi, A., Shirkhani, A., \& Chamkha, A.J. (2019). Numerical validation heat transfer of rectangular cross-section porous fins. Journal of Thermophysics and Heat Transfer, 33(3), 698-704.

[11] Hoseinzadeh, S., Heyns, P.S., Chamkha, A.J., \& Shirkhani, A. (2019). Thermal analysis of porous fins enclosure with the comparison of analytical and numerical methods. Journal of Thermal Analysis and Calorimetry, 138, 727-735.

[12] Hoseinzadeh, S., Heyns, P.S., \& Kariman, H. (2019). Numerical investigation of heat transfer of laminar and turbulent pulsating A12O3/water nanofluid flow. International Journal of Numerical Methods for Heat and Fluid Flow, 30, 1149-1166.

[13] Nithyadevi, N., Divya, V., \& Rajarathinam, M. (2017). Effect of Prandtl number on natural convection in rectangular enclosure with discrete heaters. Journal of Applied Science and Engineering, 20(2), 173-182.

[14] Patankar, S.V., \& Spalding, D.B. (1972). A calculation procedure for heat, mass and momentum transfer in three-dimensional parabolic flows. International Journal of Heat and Mass Transfer, 15(10), 1787-1806. 
[15] Saglam, M., Sarper, B., \& Aydin, O. (2017). Natural Convection Inside a Rectangular Enclosure with Two Discrete Heat Sources Placed on Its Bottom Wall. Proceedings of the 2nd World Congress on Momentum, Heat and Mass Transfer, MHMT'17, 1-8.

[16] Ambethkar, V., \& Basumatary, L.R. (2019). Numerical solutions of steady free convective flow in a rectangular region with discrete wall heat and concentration sources. Journal of Applied Mathematics and Computational Mechanics, 8(4), 5-18.

[17] Mostafa Ghiaasiaan, S. (2011). Convective Heat and Mass Transfer. Cambridge University Press.

[18] Versteeg, H.K., \& Malalasekera, W. (2010). An Introduction to Computational Fluid Dynamics: The Finite Volume Method, Pearson. 